\title{
GEDAS: A Data Management System for Data Grid Environments
}

\author{
Jaechun $\mathrm{No}^{1}$ and Hyoungwoo Park ${ }^{2}$ \\ 1 Dept. of Computer Software, \\ College of Electronics and Information Engineering, \\ Sejong University, Seoul, Korea \\ 2 Supercomputing Center, \\ Korea Institute of Science and Technology Information, \\ Daejeon city, Korea
}

\begin{abstract}
In data grid environments, many large-scale scientific experiments and simulations generate very large amounts of data in the distributed storages, spanning thousands of files and data sets. In such environments, the replication technique for the fast data sharing between the community of researchers, and the high-performance I/O for the storage and efficient data accesses on heterogeneous resources present an extremely challenging task. Several data replication techniques have been developed to support high-performance data accesses to the remotely produced scientific data. However, most of those techniques were implemented with the assumption that the data being replicated is readonly so that it would not be modified once it has been generated. Furthermore, those techniques mainly focus on measuring up the network performance, but ignoring I/O overhead incurred during the data generation and replication. We have developed a software system, called Grid Environment-based Data Management System (GEDAS), that provides a high-level, user-friendly interface, while maintaining the consistent data replicas among the grid communities. We describe the design and implementation of GEDAS and present performance results on Linux cluster.
\end{abstract}

\section{Introduction}

In data grid environments, many large-scale scientific experiments and simulations generate very large amounts of data [1,2,3, (on the order of several hundred gigabytes to terabytes) in the geographically distributed storages. Furthermore, these data are shared between the researchers and colleagues for data analysis, data visualization, and so forth. Several data replication techniques, including Globus toolkit [4,5, 6], have been developed to support high-performance data accesses to the remotely produced scientific data.

However, most of those data replication techniques were implemented with the assumption that the data being replicated is read-only so that once it has been generated would it not be modified in any grid site. Furthermore, those 
techniques mainly focus on measuring up the network performance, but ignoring the $\mathrm{I} / \mathrm{O}$ overhead incurred during the data generation and replication on the heterogeneous storages.

We have developed a software system, called Grid Environment-based Data Management System (GEDAS), that provides a high-level, user-friendly interface to share the remotely produced data among the grid communities. As a related work, we have implemented Scientific Data Manager (SDM) that combines the good features of both file I/O and databases [7,8. We, in GEDAS, extend the SDM capabilities to maintain the consistent data replicas among the grid communities and to support high-performance I/O using MPI-IO to store the real and replicated data. GEDAS interacts with database to store application-related and system-related metadata to support the integrated data replicas and highperformance I/O on the distributed resources, while taking advantage of various $\mathrm{I} / \mathrm{O}$ optimizations available in MPI-IO, such as collective I/O and noncontiguous requests, in a manner that is transparent to the user.

The rest of this paper is organized as follows. In Section 2, we discuss our goals in developing GEDAS. In Section 3, we present the design and implementation of GEDAS. Performance results on the Linux cluster located at Sejong University are presented in Section 4. We conclude in Section 5.

\section{Design Motivation}

Our main objectives in developing GEDAS were to maintain consistent data replication among the geographically distributed sites, to provide high-performance parallel I/O, to provide a high-level application programming interface (API) and to support a convenient data-retrieval capability.

- Consistent Data Replication. In order to maintain the consistently integrated data replicas among the distributed resources, GEDAS uses the version checking method that is much similar to the locking mechanism of distributed file systems [9, 10. Whenever an application running on a site modifies the replicated data, it informs the data modification to the sites that share the same data, including the owner that originally generated the data, using the version number, and thus would have them access the recently modified data.

- High-Performance I/O. To achieve high-performance I/O, GEDAS uses MPI-IO to access real data. MPI-IO, the I/O interface defined as part of the MPI-2 standard [1], is rapidly emerging as the standard, portable API for I/O in parallel applications. High-performance implementations of MPIIO, both vendor and public-domain implementation, are available for most platforms. MPI-IO is specifically designed to enable the optimizations that are critical for high-performance parallel I/O. Examples of these optimizations include collective I/O, the ability to access noncontiguous data sets, and the ability to pass hints to the implementation about access patterns, file-striping parameters, and so forth. 
- High-Level API. Our goal was to provide a high-level unified API for any kind of application (regular or irregular) while encapsulating the details of either MPI-IO or a database. The user can specify the data with a highlevel description, together with annotations, and use a similar API for data retrieval. GEDAS internally translates the user's request into appropriate MPI-IO calls, including creating MPI derived data types for noncontiguous data. GEDAS also interacts with the database when necessary, by using embedded SQL functions.

\section{$3 \quad$ Implementation Details}

We describe the design and implementation of GEDAS.

\subsection{GEDAS Metadata Structure}

Figure 1 describes an overview of GEDAS. GEDAS stores application-related metadata and system-related metadata to application_registry_table, data_registry_ table, file_registry_table, performance_registry_table, and system_registry_table. These data base tables are made for each application to provide a high-level remote data access abstraction, while supporting a transparent, user-friendly user interface.

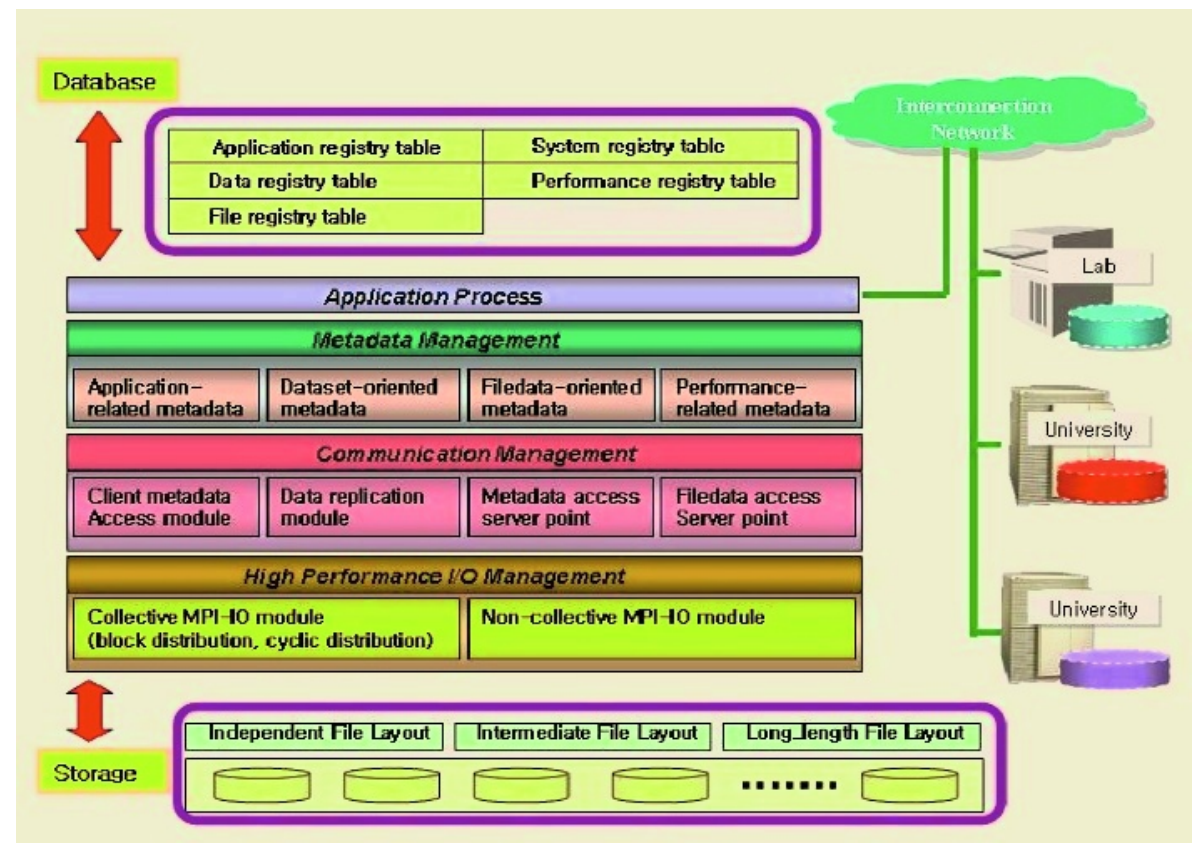

Fig. 1. GEDAS Architecture 
The data_registry_tabe includes the properties of each data set, such as data type, storage order, data access pattern, and global size. Also, it contains data replication-related metadata, such as the owner ip, replication location ip, and version number to be used in checking the replica consistency.

The file_registry_table stores a globally determined file offset denoting the starting offset of the file of each data set. GEDAS uses this information to make appropriate MPI-IO calls to access the real data. The file_registry_table also includes the physical file name to be mapped to the data set.

The system_registry_table contains the system-related metadata, including filesystem type and striping unit size where the real data is stored. This information is used to choose the optimal file layout for the real data storage.

The performance_registry_table includes the performance values evaluated using buffered $\mathrm{I} / \mathrm{O}$ and directed $\mathrm{I} / \mathrm{O}$ on each filesystem type registered in the system_registry_table. In the buffered I/O, all the data transfers between the user buffer and the storage go through the kernel buffer. On the other hand, in the directed I/O, the data transfers bypass the kernel. These values are measured using a multiple of 2 Mbytes of data size. When the data sets are accessed on a certain registered filesystem, GEDAS compares the performance values evaluated using buffered $\mathrm{I} / \mathrm{O}$ and direct $\mathrm{I} / \mathrm{O}$ to those that have been obtained using the closest data granularity in the performance_registry_table. If the direct $\mathrm{I} / \mathrm{O}$ is selected as a better I/O option, then the direct I/O flag defined in the MPI-IO is turned on to bypass the kernel buffer during the data transfer.

GEDAS provides the capability to maintain the consistent data replication among the remote resources using the data version checking. When the data requested by an application could not be found on the local storage, GEDAS connects the data owner to replicate the data to the local storage. The associated metadata stored in the application_registry_table, data_registry_table, and file_registry_table are also replicated to the local database.

If the data set needed to the application has been replicated before, then GEDAS asks the data owner for the version number associated to the data set. When the version number stored in the local database table and the one received from the data owner are the same, GEDAS reads the data replica from the local storage. Otherwise, GEDAS invalidates the data replica stored in the local storage, and then receives the data from the data owner, while updating the version number in the local database to the highest one.

\subsection{GEDAS API}

In GEDAS, users can specify groups of data sets by assigning properties to the first data set in a group and by propagating them to the other data sets belonging to the same group. The main reason for making groups of data sets is that GEDAS can then use different ways of organizing data in files, with different performance implications. For example, each data set can be written in a separate file, or the data sets of a group can be written to a single file. The properties assigned to each data set are stored in the database by invoking GEDAS_set_attributes. In case of read operation, data from a specific run can be 
retrieved by specifying attributes of the data, such as the date of the run. Also, the properties of the data sets need not be specified because GEDAS retrieves this information from the database.

The main GEDAS functions for writing and reading data are GEDAS_write and GEDAS_read. Before calling these functions, the user must provide the information necessary for GEDAS to perform I/O, such as the starting points and sizes of the subarray in each dimension in the case of block distribution, or the size of process grids and distribution arguments in each dimension in the case of cyclic distribution.

\section{Performance Evaluation}

In order to measure the performance, we used two Linux clusters located at Sejong university. Each cluster consists of four nodes having Pentium3 $866 \mathrm{MHz}$ CPU, $256 \mathrm{MB}$ of RAM, and 100Mbps of Fast Ethernet each. The operating system installed on those machines was RedHat 9.0 with Linux kernel 2.4.20-8. Each cluster uses its own PostgreSQL to store the metadata.

The performance results were obtained using the template implemented based on the three-dimensional astrophysics application, developed at the University of Chicago. The application stores block-distributed data in each dimension. In the template, we tested GEDAS library for the cyclic-distributed data as well.

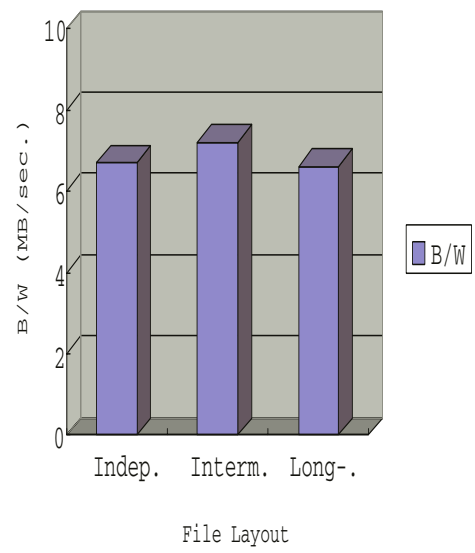

Fig. 2. Bandwidth to access the data from the remote owner in the block distribution. The accessed data is stored on the remote owner in three file layouts: independent file layout (Indep.), intermediate file layout (Interm.), and long-length file layout (Long-.)

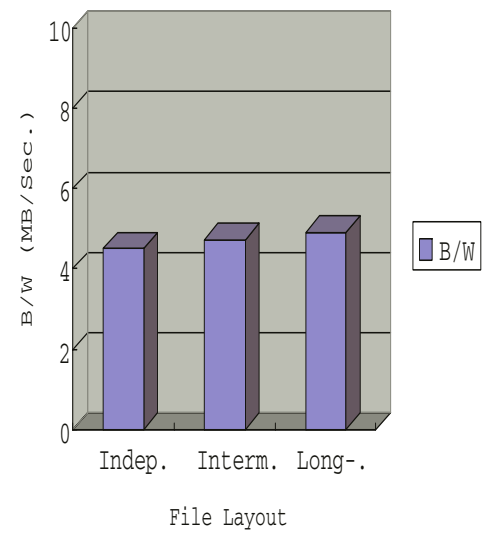

Fig. 3. Bandwidth to access the data from the remote owner in the cyclic distribution. The accessed data is stored on the remote owner in three file layouts: independent file layout (Indep.), intermediate file layout (Interm.), and long-length file layout (Long-.) 
On one of the clusters, the template has generated six floating-point arrays for data analysis, another six floating-point arrays for restart, and seven character arrays for visualization. Once those data are generated, we accessed the data from the other cluster using the GEDAS library. The template grouped the data sets into three data groups, according to the usage.

Figures 2 and 3 describe the performance results when the data sets are accessed on one cluster, while the requested data sets are stored on the other cluster in three different ways of file layout. The data access patterns tested are block distribution in Figure 2 and cyclic distribution in Figure 3. The performance values include the database overhead to access the associated metadata.

As can be seen, the bandwidth measured in the block-distributed data access pattern is higher than that measured in the cyclic data access pattern due to the smaller communication overhead incurred in the MPI-IO's collective operation. In both distributions, the independent file layout shows the least bandwidth because it generates more files than the other two file layout methods, resulting in the higher file-open and close costs.

Figures 4 and 5 demonstrate the performance results obtained to access the replica of the desired data sets in the block distribution and cyclic distribution, respectively. The replicated data is stored in the independent layout, intermediate layout, and long-length layout. When compared to Figures 2 and 3, accessing the data replica shows almost as much as twice bandwidth accessing from the remote owner because of the less communication overhead.

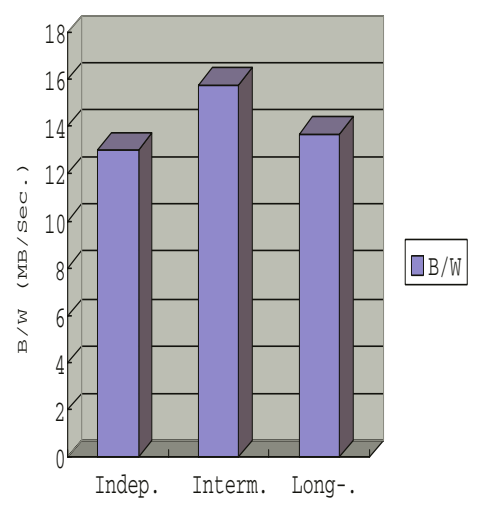

File Layout

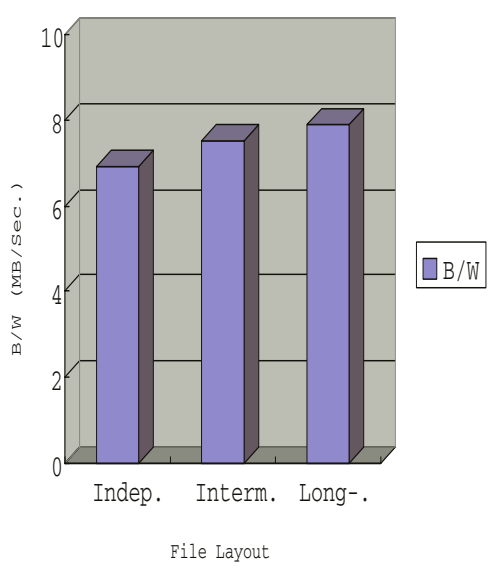

Fig. 4. Bandwidth to access the data replica in the block distribution, on top of three file layouts: independent file layout (Indep.), intermediate file layout (Interm.), and long-length file layout (Long-.)
Fig. 5. Bandwidth to access the data replica in the cyclic distribution, on top of three file layouts: independent file layout (Indep.), intermediate file layout (Interm.), and long-length file layout (Long-.) 


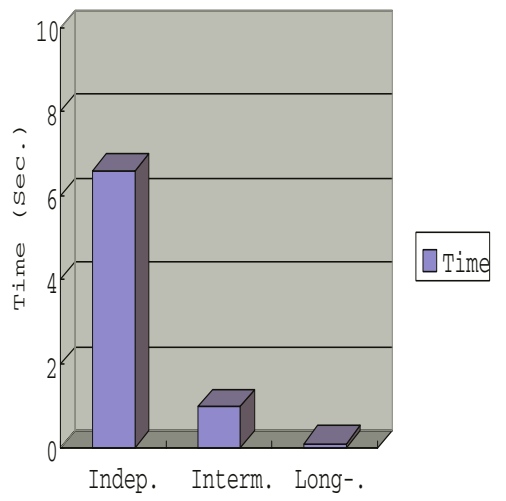

File Layout

Fig. 6. Open and close overheads of three file layouts in time

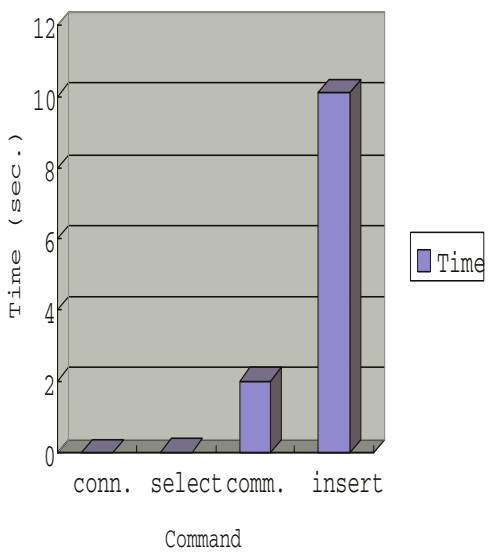

Fig. 7. Database overhead in time for the data replication

Figure [6] shows the time overhead to open and close files, on top of Linux kernel 2.4.20-8. In the template, the independent file layout produces fifty-seven files: eighteen files for data analysis, twenty-one files for data visualization, and another eighteen files for restart. The intermediate file layout generates nineteen files: six files for data analysis, another six files for restart, and seven files for data visualization. The long-length file layout generates only three files, one for each data group.

Figure 6 shows that the less files are generated to store the data sets, the less file-open and close costs are incurred on Linux kernel 2.4.20-8.

Figure 7 shows the times to access the associated metadata from the remote database necessary for replicating the desired data set to the local storage. It demonstrates that the cost for inserting the metadata received to the local database is the highest overhead in the metadata communication.

\section{Conclusion}

We have presented the design and implementation of a toolkit, called GEDAS, for high-performance scientific data management in data grid environments. GEDAS provides a simple, high-level interface and performs all necessary I/O optimizations to access and replicate the remote data transparently to the user. We also experimented with different ways of organizing data in files, called independent file layout, intermediate file layout, and long-length file layout. In general, when file-open cost on a particular file system is high, long-length file layout performs well because it minimizes the number of files created. If the file-open cost is small, the performance of the three file layouts depends on how the number and size of files affect performance on the particular file system. An 
appropriate file layout policy can thereby be chosen for a particular file system. Also, we compared the performance produced in accessing the data sets from replicas to that in accessing the data sets from the remote owner. In the future, we plan to use GEDAS with more applications and evaluate both the usability and performance.

\section{References}

1. B. Allcock, I. Foster, V. Nefedova, A. Chervenak, E. Deelman, C. Kesselman, J. Leigh, A. Sim, A. Shoshani, B. Drach, and D. Williams. High-Performance Remote Access to Climate Simulation Data: A Challenge Problem for Data Grid Technologies. SC2001, November 2001

2. R. Moore, A. Rajasekar. Data and Metadata Collections for Scientific Applications. High Performance Computing and Networking (HPCN 2001), Amsterdam, NL, June 2001

3. A. Chervenak, E. Deelman, C. Kesselman, L. Pearlman, and G. Singh. A Metadata Catalog Service for Data Intensive Applications. GriPhyN technical report, 2002

4. I. Foster, C. Kesselman, J. Nick, and S. Tuecke. The Physiology of the Grid: An Open Grid Services Architecture for Distributed Systems Integration WG, Global Grid Forum, June 22, 2002

5. A. Chervenak, I. Foster, C. Kesselman, C. Salisbury, S. Tuecke. The Data Grid: Towards an Architecture for the Distributed Management and Analysis of Large Scientific Datasets. Journal of Network and Computer Applications, 23:187-200, 2001

6. I. Foster, C. Kesselman. Globus: A Metacomputing Infrastructure Toolkit. Intl J. Supercomputer Applications, 11(2):115-128, 1997.

7. J. No, R. Thakur, and A. Choudhary. High-Performance Scientific Data Management System. Journal of Parallel and Distributed Computing, (64)4:434-447, April 2003

8. X. Shen, A. Choudhary. A Multi-Storage Resource Architecture and I/O Performance Prediction for Scientific Computing. In 9th IEEE Symposium on High Performance Distributed Computing, 2000

9. C. A. Thekkath, T. Mann, and E. K. Lee. Frangipani: A Scalable Distributed File System. In Proceedings of the Symposium on Operating Systems Principles, 1997, pages $224-237$

10. K. W. Preslan, A. P. Barry, J. E. Brassow, G. M. Erickson, E. Nygaard, C. J. Sabol, S. R. Soltis, D. C. Teigland, and M. T. O'Keefe. A 64-bit Shared Disk File System for Linux. In Proceedings of Sixteenth IEEE Mass Storage Systems Symposium Seventh NASA Goddard Conference on Mass Storage Systems \& Technologies, March 15-18, 1999

11. R. Thakur and W. Gropp. Improving the Performance of Collective Operations in MPICH. In Proceedings of the 10th European PVM/MPI Users' Group Conference (Euro PVM/MPI 2003), September 2003 